\title{
A standardized comparison of peri-operative complications after minimally invasive esophagectomy: Ivor Lewis versus McKeown.
}

\author{
Andrew M. Brown \\ Thomas Jefferson University Hospital \\ Michael J. Pucci \\ Thomas Jefferson University \\ Adam C. Berger \\ Thomas Jefferson University \\ Talar Tatarian \\ Thomas Jefferson University \\ Althanpiets an Evarastiththal works at: https://jdc.jefferson.edu/surgeryfp \\ Thomas Jefferson University \\ Part of the Surgery Commons

\section{Let us know how access to this document benefits you} \\ See next page for additional authors
}

\section{Recommended Citation}

Brown, Andrew M.; Pucci, Michael J.; Berger, Adam C.; Tatarian, Talar; Evans III, Nathaniel R.; Rosato, Ernest L.; and Palazzo, Francesco, "A standardized comparison of peri-operative complications after minimally invasive esophagectomy: Ivor Lewis versus McKeown." (2018). Department of Surgery Faculty Papers. Paper 171.

https://jdc.jefferson.edu/surgeryfp/171

This Article is brought to you for free and open access by the Jefferson Digital Commons. The Jefferson Digital Commons is a service of Thomas Jefferson University's Center for Teaching and Learning (CTL). The Commons is a showcase for Jefferson books and journals, peer-reviewed scholarly publications, unique historical collections from the University archives, and teaching tools. The Jefferson Digital Commons allows researchers and interested readers anywhere in the world to learn about and keep up to date with Jefferson scholarship. This article has been accepted for inclusion in Department of Surgery Faculty Papers by an authorized administrator of the Jefferson Digital Commons. For more information, please contact: JeffersonDigitalCommons@jefferson.edu. 
Authors

Andrew M. Brown, Michael J. Pucci, Adam C. Berger, Talar Tatarian, Nathaniel R. Evans III, Ernest L. Rosato, and Francesco Palazzo 


\title{
A Standardized Comparison of Peri-Operative Complications After Minimally Invasive Esophagectomy: Ivor Lewis vs. McKeown.
}

\author{
Andrew M Brown M.D. \\ Michael J Pucci M.D. F.A.C.S. \\ Adam C Berger M.D. F.A.C.S. \\ Talar Tatarian M.D. \\ Nathaniel R Evans III M.D. F.A.C.S. \\ Ernest L Rosato M.D. F.A.C.S. \\ Francesco Palazzo M.D. F.A.C.S. \\ Department of Surgery, Thomas Jefferson University Hospital, Philadelphia, PA, USA.
}

Running Head: Complications after esophagectomy

Author Correspondence:

Francesco Palazzo M.D. F.A.C.S

Associate Professor of Surgery

Department of Surgery

Thomas Jefferson University Hospital

1100 Walnut St. $5^{\text {th }}$ Floor

Philadelphia, PA 19107, USA

Telephone: (215) 955-1622

Fax: (215) 923-8222

Email: Francesco.Palazzo@Jefferson.edu 


\section{Abstract \\ Background}

While our institutional approach to esophageal resection for cancer has traditionally favored a minimally invasive (MI) 3-hole, McKeown esophagectomy (MIE 3-hole), during the last five years several factors have determined a shift in our practice with an increasing number of minimally invasive Ivor Lewis (MIE IL) resections being performed. We compared perioperative outcomes of the two procedures, hypothesizing that MIE IL would be less morbid in the peri-operative setting compared to MIE 3-hole.

\section{Methods}

Our institution's IRB-approved esophageal database was queried to identify all patients who underwent totally MI esophagectomy (MIE IL vs. MIE 3-hole) from June 2011 to May 2016. Patient demographics, preoperative and peri-operative data, as well as postoperative complications were compared between the two groups. Post-operative complications were analyzed using the Clavien-Dindo classification system.

Results

There were 110 patients who underwent totally MI esophagectomy (MIE IL n=49 [45\%], MIE 3-hole $n=61$ [55\%]). The majority of patients were men ( $n=91,83 \%)$ with a median age of 62.5 (range: 31-83). Preoperative risk stratifiers such as ECOG score, ASA, and Charlson Comorbidity Index were not significantly different between groups. Anastomotic leak rate was $2.0 \%$ in the MIE IL group compared to $6.6 \%$ in the MIE 3-hole group ( $\mathrm{p}=0.379)$. The rate of serious (Clavien-Dindo 3, 4 or 5) post-operative complications was significantly less in the MIE IL group (34.7 \% vs. $59.0 \%$, p=0.013). Serious pulmonary 
complications were not significantly different (16.3\% vs. $26.2 \%, p=0.251$ ) between the two groups.

Conclusions

In this cohort, totally MIE IL showed significantly less severe peri-operative morbidity than MIE 3-hole, but similar rates of serious pulmonary complications and anastomotic leaks. These findings confirm the safety of minimally invasive Ivor Lewis esophagectomies for esophageal cancer when oncologically and clinically appropriate. Minimally invasive McKeown esophagectomy remains a satisfactory and appropriate option when clinically indicated.

Key Words: Esophageal cancer, Minimally Invasive Esophagectomy, Anastomotic Leak, Ivor Lewis Esophagectomy, McKeown 3-hole Esophagectomy 


\section{Introduction}

The management of localized esophageal cancer consists of a multimodality approach that includes surgeons, as well as medical and radiation oncologists. Early stage disease can be treated with surgery alone, while locally advanced disease requires neoadjuvant chemotherapy and radiation followed by surgical management [1]. While esophagectomy, with or without neoadjuvant chemoradiotherapy, remains the cornerstone of the treatment paradigm, it has been plagued by high rates of morbidity and mortality. Perioperative morbidity rates continue to range from 26 to $41 \%[1,2,3]$.

Since the advent of minimally invasive techniques in the early 1990 s $[4,5]$, there have been improvements in short term outcomes while maintaining similar oncologic efficacy as compared to traditional open surgery $[6,7,8,9]$.

For transthoracic minimally invasive esophagectomy (MIE), there exist two distinct options: Ivor Lewis (MIE IL) and McKeown (MIE 3-hole) esophageal resections [10]. These techniques have separately been shown to be efficacious in several high-volume centers $[11,12,13]$. While the superiority of one procedure over the other continues to be matter of debate, the MIE IL approach has been favored by an increasing number of surgeons [1]. The arguments used include: the perceived decrease in morbidity derived from an anastomosis in the chest, increased recurrent laryngeal nerve injury and swallowing dysfunction with cervical incisions, and a change in the epidemiology of esophageal cancer with an increased number of adenocarcinomas of the distal esophagus and gastroesophageal junction (GEJ) $[12,14]$. However, there have been only a few attempts in the literature to evaluate peri-operative outcomes in a head to head comparison of MIE IL and MIE 3-hole $[12,15]$. In the largest series by Luketich et al. [12] in 2012, there was a 
trend towards decreased major morbidity and mortality in the MIE IL group as compared to MIE 3-hole. Two large randomized trials, the TIME trial $[16,17]$ and the MIRO trial [18] looked at open versus MIE, separately using both MIE techniques, but did not compare MIE IL to MIE 3-hole head to head.

In our institution, we have historically performed a McKeown esophagectomy but have transitioned from McKeown to Ivor Lewis in recent years. In this analysis, we review our experience with either intervention, comparing perioperative outcomes and complication rates. We hypothesized that peri-operative morbidity in Ivor Lewis MIE would be lower than McKeown MIE.

\section{Materials and Methods}

\section{Inclusion and Exclusion Criteria}

We identified patients from our Institutional Review Board-approved, prospectively maintained database of patients who underwent esophagectomy for esophageal cancer at our institution from June 2011 to May 2016 (IRB \#12D.11). Patients were included if they had undergone one of two techniques of totally minimally invasive esophagectomies: a minimally invasive Ivor Lewis esophagectomy via a laparoscopic and thoracoscopic approach or a minimally invasive McKeown esophagectomy - initially thoracoscopy, followed by laparoscopy, and cervicotomy. Patients were excluded if they underwent a hybrid surgical approach, defined as having had one of the components of the procedure (abdominal or thoracic) completed in an open fashion. No transhiatal esophagectomies were included in this analysis. 


\section{Oncologic Treatment Protocol}

All patients were preoperatively staged using a variety of imaging techniques including: contrast-enhanced CT scan of the chest/abdomen and pelvis, PET scan, and endoscopic ultrasound. Following clinical staging, those patients with greater than a T2 lesion, as well as those with positive nodes, underwent neoadjuvant chemotherapy and radiation consisting of induction radiation therapy with 4,500 cGy and either 5fluorouracil/cisplatin or 5-fluorouracil/carboplatin/paclitaxel chemotherapy with the esophageal resection scheduled an average of 6 weeks after the completion of the treatment. Those patients that were staged with under $\mathrm{T} 2$ disease, underwent primary surgical resection.

\section{Surgical Technique}

Totally minimally invasive Ivor Lewis and McKeown esophagectomies were performed as directed by surgeon preference and tumor location. This was accompanied by a standard two-field lymphadenectomy, with removal of the left gastric and celiac nodes in the abdomen, as well as the peri-esophageal and subcarinal nodes in the chest.

We have previously described our surgical technique for both MIE 3-hole and MIE IL $[7,19]$. In brief, during a MIE 3-hole, the procedure starts with the thoracoscopic mobilization of the esophagus and lymphadenectomy through a right video-assisted thoracoscopic surgery (VATS) approach, followed by laparoscopic gastric mobilization and lymphadenectomy, as well as extracorporeal gastric conduit creation, pyloromyotomy/pyloroplasty, and feeding jejunostomy placement in the supine position. Finally, a linear stapled esophagogastrostomy is performed through a left cervical incision. 
The MIE IL is begun with the patient in the supine position, and through a laparoscopic approach, key steps include: gastric mobilization, lymphadenectomy, pyloromyotomy/pyloroplasty, gastric conduit creation, and feeding jejunostomy placement [7]. The procedure is then completed through a right VATS approach, with esophageal mobilization and lymphadenectomy being performed, followed by the creation of a circularly stapled intrathoracic esophagogastric anastomosis.

\section{Pathology}

Patients included in this analysis underwent esophagectomy for adenocarcinoma or squamous cell carcinoma of the esophagus, gastroesophageal junction, or gastric cardia. The final pathologic staging was determined according to the 7th edition of the American Joint Committee on Cancer staging system [20]. A complete pathologic response was achieved when no tumor cells were identified. Completeness of the surgical resection margin was defined as: no microscopic evidence of tumor at margin (R0), microscopic evidence of tumor cells at the margin (R1), or macroscopic evidence of tumor at the surgical margin (R2). All diagnoses were confirmed by two attending pathologists.

\section{Surgical Complications and Perioperative variables}

During chart review, data collected included age, sex, body mass index (BMI), Eastern Cooperative Oncology Group (ECOG) performance status [21], American Society of Anesthesiologists Physical Status Classification (ASA) [22], Charlson Comorbidity indices (CCI) [23], initial histologic diagnosis and clinical staging, surgical technique, completeness of resection, number of lymph nodes retrieved, estimated blood loss, and operating room 
time. ECOG performance status describes a patient's level of functioning in terms of their ability to care for themselves, daily activity, and physical ability [21]. It is scored from 0 (fully active) to 5 (dead). The ASA physical status classification describes operative risk as it relates to the patient's level of systemic disease. It is scored from ASA I (normal healthy patient) to ASA VI (a declared brain dead patient, whose organs are for harvest) [22]. CCI predicts one-year mortality for a patient with a variety of conditions. A score of 1,2,3 or 6 is given to each of 22 different conditions with an age factor included to create an aggregate score, with a higher score being associated with higher rates of mortality [23].

Furthermore, post-operative complications were graded using a modification of the Clavien-Dindo (CD) scale [24]. The Clavien-Dindo classification is a scoring system based on the interventions necessary to correct a post-operative complication. These range from grade I (any complications that doesn't need management, or simple medications such as antiemetics), to grade 5 (death) [24].

Anastomotic leak was defined as purulent drainage including saliva or bile from the neck drain or cervical incision, or infection of the neck incision and confirmation of leak by a "grape juice test", contrast swallow, or endoscopic confirmation [7]. Additional postoperative data included length of stay (LOS), and complications at 90 and 180 days postoperatively.

\section{Statistical Analysis}

Descriptive statistics were computed with frequencies, means, and medians, and were stratified by the surgical approach. Quantitative analyses were performed using Student's t-tests and Fisher's Exact Tests. Statistical significance was established at 0.05. 


\section{Results}

\section{Demographics and Preoperative Factors}

There were 110 patients who underwent either MIE IL or MIE 3-hole during the time period. Of those patients, 49 (45\%) underwent an MIE IL resection, while 61 (55\%) underwent a MIE 3-hole resection. Median age, sex, and BMI were not statistically significant between the two groups. Of note, the majority of patients in both groups were male (83.6\% vs. 81.6\%). Regarding preoperative diagnosis, the MIE IL group had significantly higher stage tumors as shown in Table 1. The three measures for assessment of preoperative morbidity are shown in Table 1, including ECOG, ASA, and CCI. Only ASA scores were significantly different with the MIE 3-hole group being higher (2.92 vs. 2.73, $\mathrm{p}=0.022$ ). There was a trend towards more patients undergoing neoadjuvant treatment in the MIE IL group (79.6\%) as compared to the MIE 3-hole group (62.3\%), but this was not statistically significant $(\mathrm{p}=0.061)$.

Oncologic efficacy was evaluated in both minimally invasive procedures. The percentage of R0 resections was 94\% (MIE 3-hole) and 100\%, (MIE IL) and were similar, as were total lymph node harvested between groups. This is shown in Table 2 . There was a trend towards higher rates of positive lymph nodes harvested in the MIE IL group, but this was not statistically significant. Neither estimated blood loss, nor operating room times were significantly different between the two groups. Median length of hospital stay was also similar between groups. 
Peri-operative complications were classified by specific complication category, and graded according to Clavien-Dindo. As shown in Table 3, the anastomotic leak rate was $6.6 \%$ in the MIE 3-hole group, and 2.0\% in the MIE IL group ( $\mathrm{p}=0.379)$. Similarly, rates of all pulmonary and cardiac complications were not statistically significant between groups $(\mathrm{p}=0.310$ and $\mathrm{p}=0.218$ respectively). Rates of complications in all other physiologic categories were not significantly different, including vocal cord paresis.

Rates of serious (CD grade $\geq 3$ ) complications were analyzed between groups. The rates of all serious complications was significantly higher in MIE 3-hole compared to MIE IL (59.0 \% vs. 34.7\%, p=0.013). Serious anastomotic leaks, pulmonary and cardiac complications, as well as infectious complications were not statistically different, as shown in Table 4.

\section{Follow-up}

Mortality within 30 days was 1.6\% (MIE 3-hole) vs. 6.1\% (MIE IL), p=0.322.

Readmission rates at 30 days were low in both groups, at 3.3\% and 2.0\%. Median follow-up was 21.6 months (MIE 3-hole) and 9.0 months (MIE IL). The rates of serious complications at 90 and 180 days were analyzed. Rates were not significantly different at either time point. Short-term follow-up information is shown in Table 5. Of note, serious anastomotic stricture rates were not different between groups at 180 days postoperatively. There were no significant differences between the two groups in either overall survival (83.6\% in MIE 3-hole vs. 79.6\% MIE IL), or disease-free survival (73.8\% vs. 63.2\% respectively, $p=0.300$ ).

\section{Discussion}


We have previously reported on the safety and oncologic efficacy of MIE in esophageal cancer [7]. This initial experience was primarily with patients who had undergone MIE 3-hole, but over the course of several years, we have modified our practice to include MIE IL and MIE 3-hole. The trend from more MIE 3-hole to MIE IL has been the result of the changing epidemiology of esophageal cancer, the increasing numbers of gastroesophageal junction adenocarcinoma, and was stimulated by surgeon's preference within our group due to the perception of potentially fewer complications. A major reason for the change in surgical technique stems from large cohort studies from high volume centers showing potential improvement with MIE IL $[12,25]$. The group led by Luketich et al. described their transition from a MIE 3-hole to an MIE IL approach in large part due to the morbidity associated with the cervical incision. They describe higher incidences of anastomotic leak, anastomotic stricture, recurrent laryngeal nerve injury, and pharyngoesophageal swallowing dysfunction following a MIE 3-hole approach. Their group virtually eliminated recurrent laryngeal nerve injury, while limiting the length of the gastric conduit required, and allowing for a more aggressive gastric resection margin by switching to an MIE IL resection. That data has informed our group, and encouraged a transition to minimally invasive Ivor Lewis esophagectomies for distal esophageal, and GEJ tumors. This trend is reflected elsewhere in the literature with other surgeons having used MIE IL resections for not only tumors of gastroesophageal junction, but also esophageal tumors of the distal third of the esophagus [26]. In our institution, currently a MIE IL resection is offered in all cases of esophageal cancer when clear margins can be obtained. While the debate over the superiority of either approach (MIE IL vs MIE 3-hole) continues, 
we sought to review our current experience with these two techniques using a standardized complications grading system [24].

In this report, the two cohorts that were compared appeared to be well matched based on preoperative criteria. Regarding our ASA scores, the MIE 3-hole group has significantly higher scores ( 2.92 vs. $2.73, \mathrm{p}=0.022$ ). While this could indicate that the MIE 3 hole patients were sicker preoperatively, this scoring system is highly operator dependent, and additionally, a 0.2 difference of mean score is likely not to be clinically significant. A statistically significant difference was present in the preoperative stage between MIE IL and 3-hole MIE. This may be the result of selection bias as patients with more advanced disease could be allocated to MIE IL. Also, there was a trend, ultimately not statistically significant, to more of the MIE IL patients having undergone neoadjuvant therapy which is likely related to the higher preoperative stage.

Perioperative outcomes and oncologic efficacy, measured by completeness of resection and number of LN harvested, were similar between the two groups. Furthermore, the results show a significant difference in the totally minimally invasive Ivor Lewis esophagectomy group having had significantly less severe complications (34.7\%) than minimally invasive McKeown esophagectomy (59.0\%). Looking at a breakdown of all complications between the two groups, there were similar rates of serious pulmonary complications, cardiac complications, anastomotic leaks, and vocal cord paresis. Oncologic efficacy, measured by completeness of resection and number of LN harvested, was similar between the two groups. Interestingly, our patients who underwent MIE IL resection had fewer serious complications while having a significantly higher preoperative clinical stage. Notably, the incidence of GI related complications, including delayed gastric emptying and 
esophago-gastric anastomotic strictures among others, was not found to be statistically significant in this cohort. In a previous analysis from our group looking at the number of endoscopic interventions for gastrointestinal symptoms after MIE, we did identify a marked difference in favor of MIE IL [27].

These data confirm the available evidence $[12,14]$ and provide results using a standardized assessment with preoperatively clearly defined characteristics, and postoperative severity of complications using a validated grading system (CD).

Luketich [11] et al. series of 222 patients who underwent MIE 3-hole showed anastomotic leak rates of $11.7 \%$, and rates of "major complications" of 32\%. Our patient series of MIE 3-hole compares favorably in terms of anastomotic leak rate and unfavorably for overall major complications. In a separate series, the same authors reported a 4\% anastomotic leak rate with MIE IL, and a rate of "major morbidity" of 18\% [12]. Our anastomotic leak rate compares favorably, while our rates of major complications are higher. In a very recent series, Straatman [28] et al showed that in 282 patients who underwent MIE IL, there was a 15.2\% anastomotic leak rate, 13.1\% pulmonary complication rate, and $4.3 \%$ cardiovascular complication rate. Our data shows higher rates of cardio-pulmonary complications, but anastomotic leak rate superiority in a smaller series. These results could be due to perioperative interventions (related to anesthesia, patient positioning, length of surgical intervention, or postoperative pulmonary care) that will require further analysis on our part. When comparing to the Straatman et al series, our operative times of greater than 600 minutes differs greatly from their reported mean of 333 minutes [28]. Some of that difference may be due to differences in timing methodology between institutions. At our institution, anesthesia time, as well as positioning and any 
pre-incision procedures are including in the operative length. Additionally, there are often delays in repositioning between the abdominal and thoracic portions of the operation that are being addressed.

Pulmonary complications in particular can provide significant morbidity following esophagectomy. Regarding our data, we had rates of 26.5\% (MIE IL) vs. 36.1\% (MIE 3-hole) in terms of overall complications including all Clavien-Dindo grades. With respect to serious complications (CD grade greater than or equal to three), there were rates of $16.3 \%$ (MIE IL) and 26.2\% (MIE 3-hole). By comparison, in the TIME trial [16], the authors reported all pulmonary complication rates of $12 \%$ in the MIE 3-hole group. Importantly, these patients underwent the thoracoscopic portion of their procedure in the prone position, which may positively impact their pulmonary complication rates. In the MIRO trial [18], the authors report major pulmonary complication rates of $17.7 \%$ in their MIE IL which is comparable to our data. With regards to one-year overall, and disease-free survival, there was no significant difference between our two groups, but the follow-up is very short. The aim of this study was focused on post-operative complications between the two techniques, and survival will be addressed further in subsequent analyses.

The results reported here favor a trend towards performing a minimally invasive Ivor Lewis esophagectomy for esophageal cancer when clinically and oncologically appropriate.

This analysis obviously should be interpreted with caution due to limitations intrinsic to the methodological design that need to be acknowledged. First, this is a retrospective review of a relatively small sample size. There was no randomization to surgical technique, and in general the choice of operation was based on surgeon 
preference. In recent years, patients with more distally located tumors were typically offered an MIE IL resection. While this lack of randomization is somewhat limiting, our patient cohorts appear to be well matched in terms of preoperative factors including ECOG score and CCI, as well as age, sex, and BMI. This blunts some of the allocation bias associated with a lack of randomization. Importantly, our MIE IL patients have significantly higher preoperative clinical stages while still maintaining equal, and sometimes better complication rates.

Second, data accrued from the MIE 3-hole group, while spanning all five years, is more heavily weighted towards the beginning part of the study period. Even in that short amount of time, there have been advances in perioperative anesthetic and critical care management that may have impacted patient outcomes. These may sway complication numbers in favor of MIE IL. Finally, our analysis comparing totally minimally invasive options for esophagectomy exclude transhiatal MIE. This is not a procedure currently performed at our institution, and conclusions regarding peri-operative complications of MIE should be limited to comparisons between MIE IL and MIE 3-hole. The technical challenges associated with a transhiatal approach have been well documented, and have encouraged a transition to transthoracic approaches [29].

To our knowledge this is the first report to compare, in a standardized fashion, a cohort of contemporary patients undergoing totally MIE with the two approaches of IL and McKeown. We measured accepted preoperative characteristics, and utilized a validated post-operative complications grading system. These data support minimally invasive Ivor Lewis esophagectomy as a safer procedure for the treatment of esophageal cancer when compared to minimally invasive McKeown esophagectomy. The results also confirm that 
minimally invasive McKeown esophagectomy, when clinically indicated, maintains a satisfactory safety profile. Additionally, while pulmonary complication rates compared favorably to some published reports on MIE, there is certainly an opportunity to improve on the current data by considering additional perioperative pulmonary interventions. 


\section{Disclosures}

Drs. Andrew M Brown, Michael J Pucci, Adam C Berger, Talar Tatarian, Nathaniel R Evans

III, Ernest L Rosato, and Francesco Palazzo have no conflicts of interest or financial ties to disclose. 


\section{References}

1. D'Journo XB, Thomas PA (2014), Current management of esophageal cancer. J Thorac Dis, 6:S253-264.

2. Enzinger PC, Mayer RJ (2003), Esophageal cancer. N Engl J Med, 349:2241-2252.

3. Sihag S, Kosinski AS, Gaissert HA, Wright CD, Schipper PH (2016), Minimally Invasive Versus Open Esophagectomy for Esophageal Cancer: A Comparison of Early Surgical Outcomes From The Society of Thoracic Surgeons National Database. Ann Thorac Surg, 101:1281-1288; discussion 1288-1289.

4. Dallemagne B, Weerts JM, Jehaes C, Markiewicz S, Lombard R (1991), Laparoscopic Nissen Fundoplication: preliminary report, Surg Laparosc Endosc, 1:138-143.

5. Cuschieri A (1994), Thoracoscopic subtotal oesophagectomy. Endosc Surg Allied Technol, 2:21-25.

6. Pennathur A, Luketich JD (2012), Minimally invasive esophagectomy: short-term outcomes appear comparable to open esophagectomy. Ann Surg, 255:206-207.

7. Palazzo F, Rosato EL, Chaudhary A, Evans NR, Sendecki JA, Keith S, Chojnacki KA, Yeo CJ, Berger AC (2015), Minimally invasive esophagectomy provides significant survival advantage compared with open or hybrid esophagectomy for patients with cancers of the esophagus and gastroesophageal junction. J Am Coll Surg, 220:672-679.

8. Singh RK, Pham TH, Diggs BS, Perkins S, Hunter JG (2011), Minimally invasive esophagectomy provides equivalent oncologic outcomes to open esophagectomy for locally advanced (stage II or III) esophageal carcinoma. Arch Surg, 146:711-714. 
9. Berger AC, Bloomenthal A, Weksler B, Evans N, Chojnacki KA, Yeo CJ, Rosato EL (2011), Oncologic efficacy is not compromised, and may be improved with minimally invasive esophagectomy. J Am Coll Surg, 212:560-566; discussion 566-568.

10. Giugliano DN, Berger AC, Rosato EL, Palazzo F (2016), Total minimally invasive esophagectomy for esophageal cancer: approaches and outcomes. Langenbecks Arch Surg, 401:747-756.

11. Luketich JD, Alvelo-Rivera M, Buenaventura PO, Christie NA, McCaughan JS, Litle VR, Schauer PR, Close JM, Fernando HC (2003), Minimally invasive esophagectomy: outcomes in 222 patients. Ann Surg, 238:486-494; discussion 494-495.

12. Luketich JD, Pennathur A, Awais O, Levy RM, Keeley S, Shende M, Christie NA, Weksler B, Landreneau RJ, Abbas G, Schuchert MJ, Nason KS (2012), Outcomes after minimally invasive esophagectomy: review of over 1000 patients. Ann Surg, 256:95-103.

13. Palanivelu C, Prakash A, Senthilkumar R, Senthilnathan P, Parthasarathi R, Rajan PS, Venkatachlam S (2006), Minimally invasive esophagectomy: thoracoscopic mobilization of the esophagus and mediastinal lymphadenectomy in prone position--experience of 130 patients. J Am Coll Surg, 203:7-16.

14. Tapias LF, Mathisen DJ, Wright CD, Wain JC, Gaissert HA, Muniappan A, Lanuti, M, Donahue DM, Morse CR (2016), Outcomes With Open and Minimally Invasive Ivor Lewis Esophagectomy After Neoadjuvant Therapy. Ann Thorac Surg, 101:1097-1103.

15. Zhai C, Liu Y, Li W, Xu T, Yang G, Lu H, Hu D (2015), A comparison of short-term outcomes between Ivor-Lewis and McKeown minimally invasive esophagectomy. J Thorac Dis, 7:2352-2358. 
16. Biere SS, van Berge Henegouwen MI, Maas KW, Bonavina L, Rosman C, Garcia JR, Gisbertz SS, Klinkenbijl JH, Hollmann MW, de Lange ES, Bonjer HJ, van der Peet DL, Cuesta MA (2012), Minimally invasive versus open oesophagectomy for patients with oesphageal cancer: a multicenter, open-label, randomized controlled trial. Lancet, 379:1887-1892.

17. Straatman J, van der Wielen N, Cuesta MA, Daams F, Roig Garcia J, Bonavina L, Rosman C, van Berge Henegouwen MI, Gisbertz SS, van der Peet DL (2017), Minimally Invasive Versus Open Esophageal Resection: Three-year Follow-up of the Previously Reported Randomized Controlled Trial: the TIME Trial. Ann Surg, [epub ahead of print].

18. Briez N, Piessen G, Bonnetain F, Brigand C, Carrere N, Collet D, Doddoli C, Flamein R, Mabrut JY, Meunier B, Msika S, Perniceni T, Peschaud F, Prudhomme F, Triboulet JP, Mariette C (2011), Open versus laparoscopically-assisted oesophagectomy for cancer: a multicenter randomized controlled phase III trial - the MIRO trial. BMC Cancer, 11:310. 19. Palazzo F, Evans NR, Rosato EL (2013), Minimally invasive esophagectomy with extracorporeal gastric conduit creation--how I do it. J Gastrointest Surg, 17:1683-1688. 20. Edge SB, Byrd DR, Compton CC, Fritz AG, Greene FL, Trotti A (2009) AJCC Cancer Staging Manual, Springer-Verlag, New York, NY.

21. Oken MM, Creech RH, Tormey DC, Horton J, Davis TE, McFadden ET, Carbone PP (1982), Toxicity and response criteria of the Eastern Cooperative Oncology Group. Am J Clin Oncol, 5:649-655.

22. Cullen DJ, Apolone G, Greenfield S, Guadagnoli E, Cleary P (1994), ASA Physical Status and age predict morbidity after three surgical procedures. Ann Surg, 220:3-9. 
23. Charlson ME, Pompei P, Ales KL, MacKenzie CR (1987), A new method of classifying prognostic comorbidity in longitudinal studies: development and validation. J Chronic Dis, 40:373-383.

24. Dindo D, Demartines N, Clavien PA (2004), Classification of surgical complications: a new proposal with evaluation in a cohort of 6336 patients and results of a survey. Ann Surg, 240:205-213.

25. Levy RM, Trivedi D, Luketich JD (2012), Minimally Invasive Esophagectomy. Surg Clin N Am, 92:1265-1285

26. Sutton CD, White SA, Marshall LJ, Berry DP, Veitch PS (2002), Endoscopic-assisted intrathoracic oesophagogastrostomy without thoracotomy for tumours of the lower oesophagus and cardia. Eur J Surg Oncol, 28:46-48.

27. Giugliano DN, Berger AC, Meidl H, Pucci MJ, Rosato EL, Keith SW, Evans NR, Palazzo F (2017) Do Intraoperative Pyloric Interventions Predict the Need for Postoperative Endoscopic Interventions after Minimally Invasive Esophagectomy? Dis Esophagus, [in press].

28. Straatman J, van der Wielen N, Nieuwenhuijzen GA, Rosman C, Roig J, Scheepers JJ, Cuesta MA, Luyer MD, van Berge Henegouwen MI, van Workum F, Gisbertz SS, van der Peet DL (2017), Techniques and short-term outcomes for total minimally invasive Ivor Lewis esophageal resection in distal esophageal and gastroesophageal junction cancers: pooled data from six European centers. Surg Endosc, 31:119-126.

29. Zhang J, Wang R, Liu S, Luketich JD, Chen S, Chen H, Schuchert MJ (2012), Refinement of Minimally Invasive Esophagectomy Techniques After 15 Years of Experience. J Gastrointest Surg, 16:1768-1774. 